\title{
PSEUDO-CHERN CLASSES OF AN ALMOST PSEÚUO-HERMITIAN MANIFOLD
}

\author{
YASUO MATSUSHITA
}

\begin{abstract}
For an almost pseudo-Hermitian manifold, pseudo-Chern classes are defined on its complexified tangent bundle with the pseudo-Hermitian structure as represented by certain $\operatorname{ad}(U(p, q))$-invariant forms on the manifold. It is shown that such a manifold always admits an almost Hermitian structure, and hence that Chern classes are also defined on the complexified tangent bundle with such an almost Hermitian structure. A relation between the pseudo-Chern classes and the Chern classes is established. From the relation, the pseudo-Chern classes are considered as the characteristic classes which measure how the almost pseudo-Hermitian structure deviates from an almost Hermitian structure.
\end{abstract}

1. Introduction. The study of characteristic classes for pseudo-Riemannian manifolds started with the famous works of Avez $[\mathbf{A}]$ and Chern $[\mathbf{C}]$ concerning the generalized Gauss-Bonnet formula. These works assert that the Euler characteristic class of a pseudo-Riemannian vector bundle can be determined by its pseudo-Riemannian structure. The author's recent paper [M1] noted that for a pseudo-Riemannian manifold its pseudo-Pontrjagin class represented by a certain $\operatorname{ad}\left(S O_{0}(p, q)\right)$-invariant polynomial function of the pseudo-Riemannian curvature tensor coincides with the Pontrjagin class of the manifold. It should be mentioned that Borel [B] has treated these issues for pseudo-Riemannian mainfolds, dealing also with the Chern classes for pseudo-Hermitian manifolds. In $[\mathbf{B}]$, Borel asserted that these characteristic classes corresponding to the groups $S O(p+q)$ in the real case and $U(p+q)$ in the complex case can be obtained from the curvature forms in the bundles with the groups $S O_{0}(p, q)$ and $U(p, q)$, respectively.

The purpose of this paper is to show that in the complex case we can construct analogues of the Chern classes for a pseudo-Hermitian vector bundle which coincide with the Chern classes only if the negative part of the bundle makes no contribution to the characteristic classes. We shall call these characteristic classes the pseudoChern classes.

In this paper, we deal mainly with an almost pseudo-Hermitian manifold. Generalizing Gray's formula [G, Theorem 9.1], we construct pseudo-Chern classes on the complexified tangent bundle of such a manifold as represented by certain $\operatorname{ad}(U(p, q))$-invariant curvature polynomials. The result obtained in this paper are easily applicable to every complex vector bundle with a pseudo-Hermitian structure.

It is shown that every almost pseudo-Hermitian manifold admits an almost Hermitian structure, and hence that the Chern classes are also defined in the sense of

Received by the editors November 27, 1985 and, in revised form March 11, 1986.

1980 Mathematics Subject Classification. Primary 57R20, 53C15, 53C50, 53C55. 
Gray $[\mathbf{G}]$ on its complexified tangent bundle with such an almost Hermitian structure. It is crucial to recognize that the almost pseudo-Hermitian tangent bundle reduces to a Whitney sum of two tangent subbundles, and that such a splitting is compatible with the almost pseudo-Hermitian structure and also with the almost Hermitian structure on the manifold. Therefore, we can compare the pseudo-Chern classes with the Chern classes for the Whitney sum, and hence obtain a certain relation between them. From the relation, the pseudo-Chern classes are considered as the characteristic classes which measure how the almost pseudo-Hermitian structure deviates from an almost Hermitian structure.

The paper is organized as follows. $\S 2$ covers the preliminaries for treating an almost pseudo-Hermitian manifold. We define the pseudo-Chern classes for such a manifold in $\S 3$. We show in $\S 4$ that every almost pseudo-Hermitian manifold admits an almost Hermitian structure, and that its Chern classes are defined naturally. In $\S 5$, we show that the almost pseudo-Hermitian tangent bundle can be reduced to a Whitney sum of two almost Hermitian tangent subbundles. In $\S 6$, we state the main result concerning a relation between the pseudo-Chern classes and the Chern classes and discuss some other important results. We end the paper with some remarks.

2. Almost pseudo-Hermitian manifolds. Throughout this paper, by a manifold we mean a compact orientable $C^{\infty}$-differentiable manifold.

Let $(M, J,\langle\rangle$,$) be a 2 n$-dimensional almost pseudo-Hermitian manifold with an almost complex structure $J$, a $J$-invariant pseudo-Riemannian metric $\langle$,$\rangle of$ signature

$$
(+\underset{2 p}{\cdots}+-\underset{2 q}{-\cdots}), \quad 2 p \geq 2 q, 2 p+2 q=2 n,
$$

and the pseudo-Riemannian connection $\hat{\nabla}$. The metric $\langle$,$\rangle is called the pseudo-$ Hermitian metric on $M$ with the property

$$
\langle J X, J Y\rangle=\langle X, Y\rangle,
$$

where $X, Y \in \mathfrak{X}(M)$, the algebra of $C^{\infty}$ vector fields on $M$. The tangent bundle $\pi: \xi \rightarrow M$ of $M$ has pseudo-unitary group $U(p, q)$ as the structure group, where $U(p, q)$ is considered as a subgroup of the pseudo-orthogonal group $O(2 p, 2 q)$.

We can choose a local pseudo-orthonormal frame field on $M$ as

$$
\left\{E_{1}, J E_{1}, \ldots, E_{p}, J E_{p}, E_{p+1}, J E_{p+1}, \ldots, E_{n}, J E_{n}\right\}
$$

such that for $i, j=1, \ldots, n$,

$$
\left\langle E_{i}, E_{j}\right\rangle=\left\langle J E_{i}, J E_{j}\right\rangle=\varepsilon_{i} \delta_{i j}, \quad\left\langle E_{i}, J E_{j}\right\rangle=0,
$$

where $\delta_{i j}$ is the Kronecker delta, and $\varepsilon_{i}=+1$ for $1 \leq i \leq p, \varepsilon_{i}=-1$ for $p+1 \leq$ $i \leq n$.

We now consider the complexification $\xi \otimes \mathbf{C}$ of the tangent bundle $\xi$ of $M$. There are $p+q(=n)$ local complex vector fields $V_{1}, \ldots, V_{p}, W_{1}, \ldots, W_{q}$ on $\xi \otimes \mathbf{C}$ such that

$$
\left\{V_{1}, \ldots, V_{p}, W_{1}, \ldots, W_{q}, \bar{V}_{1}, \ldots, \bar{V}_{p}, \bar{W}_{1}, \ldots, \bar{W}_{q}\right\}
$$

is a local basis field for $\xi \otimes \mathbf{C}$, where the bar indicates the complex conjugation. The metric $\langle$,$\rangle can be extended uniquely to a complex symmetric bilinear form,$ 
also denoted by $\langle$,$\rangle , with the properties$

$$
\begin{gathered}
\left\langle V_{a}, \bar{V}_{b}\right\rangle=\delta_{a b}, \quad\left\langle W_{\alpha}, \bar{W}_{\beta}\right\rangle=-\delta_{\alpha \beta}, \\
\left\langle V_{a}, W_{\alpha}\right\rangle=0, \quad\left\langle V_{a}, \bar{W}_{\alpha}\right\rangle=0, \quad\left\langle\bar{V}_{a}, W_{\alpha}\right\rangle=0,
\end{gathered}
$$

for $a, b=1, \ldots, p$ and $\alpha, \beta=1, \ldots, q$. Such a basis can be expressed in terms of a suitable basis $\left\{E_{i}, J E_{j}\right\}$ as follows:

$$
\begin{array}{ll}
V_{a}=\left(E_{a}-\sqrt{-1} J E_{a}\right) / \sqrt{2}, & a=1, \ldots, p \\
W_{\alpha}=\left(E_{p+\alpha}-\sqrt{-1} J E_{p+\alpha}\right) / \sqrt{2}, & \alpha=1, \ldots, q .
\end{array}
$$

Note that $V_{a}, W_{\alpha}$ (resp. $\bar{V}_{a}, \bar{W}_{\alpha}$ ) are the $\sqrt{-1}$ (resp. $-\sqrt{-1}$ ) eigenvectors of $J$.

The pseudo-Riernannian curvature tensor $\hat{R}$ is defined by

$$
\hat{R}(X, Y) Z=\left[\hat{\nabla}_{X}, \hat{\nabla}_{Y}\right] Z-\hat{\nabla}_{[X, Y]} Z \quad \text { for } X, Y, Z \in \mathfrak{X}(M) .
$$

On the basis of Gray's analysis [G, Theorem 9.1] and associated with the pseudoRiemannian connection $\hat{\nabla}$, we define a new connection $\hat{D}$ on the tangent bundle $\xi$ of $M$ as follows:

$$
\hat{D}_{X} Y=\frac{1}{2}\left(\hat{\nabla}_{X} Y-J \hat{\nabla}_{X} J Y\right) .
$$

If $\hat{\nabla} J=0$, then $\hat{D}$ coincides with $\hat{\nabla}$. We denote by $\hat{S}$ the new pseudo-curvature tensor of the connection $\hat{D}$ :

$$
\hat{S}(X, Y) Z=\left[\hat{D}_{X}, \hat{D}_{Y}\right] Z-\hat{D}_{[X, Y]} Z \quad \text { for } X, Y, Z \in \mathfrak{X}(M) \text {. }
$$

Associated with $\hat{S}$, we obtain a complexified pseudo-curvature 2 -form $\hat{\Phi}=\left[\hat{\Phi}_{i j}\right]$ of type $(0,2)$ defined by

$$
\hat{\Phi}_{i j}(X, Y)=\left\langle\hat{S}_{X Y} E_{i}, E_{j}\right\rangle-\sqrt{-1}\left\langle\hat{S}_{X Y} E_{i}, J E_{j}\right\rangle \quad \text { for } X, Y \in \mathfrak{X}(M) \otimes \mathbf{C} .
$$

$\hat{\Phi}$ is written as an $n \times n$ skew-Hermitian matrix;

$$
{ }^{t} \hat{\Phi}+\overline{\hat{\Phi}}=0 \text {. }
$$

The pseudo-curvature 2-form of type $(1,1)$, denoted by $\hat{\Phi}^{\prime}\left(=\left[\hat{\Phi}^{\prime i}\right]\right)$, is related to $\hat{\Phi}$ by the formula

$$
\hat{\Phi}^{\prime}=I_{p, q} \hat{\Phi}, \quad I_{p, q}=\left[\begin{array}{cc}
I_{p} & 0 \\
0 & -I_{q}
\end{array}\right],
$$

where $I_{k}=\operatorname{diag}[+1, \ldots,+1](+1: k$ times $)$. Then, $\hat{\Phi}^{\prime}$ satisfies

$$
{ }^{t} \hat{\Phi}^{\prime} I_{p, q}+I_{p, q} \overline{\hat{\Phi}^{\prime}}=0 .
$$

The pseudo-unitary group $U(p, q)$ acts on $\hat{\Phi}$ and $\hat{\Phi}^{\prime}$ in the following ways, respectively:

$$
\begin{aligned}
\hat{\Phi} & \mapsto \hat{\Psi}={ }^{t} g \hat{\Phi} \bar{g}, \\
\hat{\Phi}^{\prime} & \mapsto \hat{\Psi}^{\prime}=g^{-1} \hat{\Phi}^{\prime} g,
\end{aligned}
$$

where $g \in U(p, q)$. If the structure group is a unitary group $U(n)(p=n, q=0)$, i.e., the manifold is almost Hermitian, then the complexified curvature 2-form of type $(0,2)$ and that of type $(1,1)$ coincide with each other and are written as a skew-Hermitian $n \times n$ matrix. 
3. Pseudo-Chern classes. Let $M_{n}(\mathbf{C})$ denote the algebra of all complex $n \times n$ matrices. Define a function $\hat{f}: R \times M_{n}(\mathbf{C}) \rightarrow \mathbf{C}$ by

$$
\hat{f}(\lambda, A)=(-1)^{q} \operatorname{det}\left(\lambda I_{p, q}-(2 \pi \sqrt{-1})^{-1} A\right) .
$$

Concerning this function, we have

Proposition 1. $\hat{f}(\lambda, \hat{\Phi})$ is invarant under the actions of $U(p, q)$.

PROOF. The proof is similar to the case of the pseudo-Pontrjagin classes (cf. [M1, Lemma]. For the pseudo-unitary group, we know that

$$
g \in U(p, q) \longleftrightarrow{ }^{t} g I_{p, q} \bar{g}=I_{p, q} .
$$

The group $U(p, q)$ acts on $\hat{\Phi}$ according to (1a) and hence

$$
\begin{aligned}
\hat{f}(\lambda, \hat{\Phi}) \mapsto \hat{f}(\lambda, \hat{\Psi}) & =\hat{f}\left(\lambda,{ }^{t} g \hat{\Phi} \bar{g}\right) \\
& =(-1)^{q} \operatorname{det}\left(\lambda I_{p, q}-(2 \pi \sqrt{-1})^{-1}{ }^{t} g \hat{\Phi} \bar{g}\right) \\
& =(-1)^{q} \operatorname{det}\left(\lambda^{t} g I_{p, q} \bar{g}-(2 \pi \sqrt{-1})^{-1} t g \hat{\Phi} \bar{g}\right) \\
& =(-1)^{q} \operatorname{det}\left({ }^{t} g\left(\lambda I_{p, q}-(2 \pi \sqrt{-1})^{-1} \hat{\Phi}\right) \bar{g}\right. \\
& =(-1)^{q} \operatorname{det}\left(\lambda I_{p, q}-(2 \pi \sqrt{-1})^{-1} \hat{\Phi}\right) \\
& =\hat{f}(\lambda, \hat{\Phi}) .
\end{aligned}
$$

This completes the proof.

Associated with the function $\hat{f}(\lambda, A)$ we define $\operatorname{ad}(U(p, q))$-invariant polynomial functions $\hat{f}_{0}, \hat{f}_{1}, \ldots, \hat{f}_{n}$ on the Lie algebra $u(p, q)$ by

$$
\hat{f}(\lambda, A)=\sum_{k=0}^{n} \hat{f}_{k}(A) \lambda^{n-k} \quad \text { for } A \in u(p, q) .
$$

For a $k$ th function $\hat{f}_{k}$ there exists a unique closed $2 k$-form $\gamma_{k}$ on $M$ such that $\pi^{*}\left(\gamma_{k}\right)=\hat{f}_{k}(\hat{\Phi})$.

We now denote by $\hat{c}_{k}(\xi \otimes \mathbf{C})$ the class of the $2 k$ th de Rham cohomology ring $H^{2 k}(M ; R)$ represented by $\gamma_{k}$.

DEFINITION 2. The kth pseudo-Chern class of $(M, J,\langle\rangle$,$) , denoted by \hat{c}_{k}(M)$, is defined to be the class $\hat{c}_{k}(\xi \otimes \mathbf{C})$ of the complexified pseudo-Hermitian bundle $\xi \otimes \mathbf{C}$ over $(M, J,\langle\rangle$,$) . Moreover, we put \hat{c}(M)=\hat{c}(\xi \otimes \mathbf{C})$ as

$$
\hat{c}(M)=1+\hat{c}_{1}(M)+\hat{c}_{2}(M)+\cdots+\hat{c}_{n}(M) \in H^{\star}(M ; R),
$$

and call it the total pseudo-Chern class of $(M, J,\langle\rangle$,$) . We can further define the$ pseudo-Chern character of $(M, J,\langle\rangle$,$) .$

DEFinition 3. The pseudo-Chern character of $(M, J,\langle\rangle$,$) , denoted by \widehat{c h}(M)$, is defined to be the characteristic class represented by the following $\operatorname{ad}(U(p, q))$ invariant polynomial

$$
\operatorname{trace}\left(\exp \left(-\hat{\Phi}^{\prime} / 2 \pi \sqrt{-1}\right)\right)=\operatorname{trace}\left(\exp \left(-I_{p, q} \hat{\Phi} / 2 \pi \sqrt{-1}\right)\right)
$$

REMARK. It is to be noted that a polynomial trace $(\exp (-\hat{\Phi} / 2 \pi \sqrt{-1}))$ is not adjoint invariant by $U(p, q)$, because trace for a matrix of type $(0,2)$ does not give rise to an invariant quantity by the structure group, but for that of type $(1,1)$. 
4. Chern classes. For an almost pseudo-Hermitian manifold $(M, J,\langle\rangle$,$) , if$ we forget the pseudo-Hermitian metric $\langle$,$\rangle , then we obtain an almost complex$ manifold $(M, J)$. It is known (cf. [K-N, Chapter IX, Proposition 4.1]) that such a pair $(M, J)$ admits a $J$-invariant positive definite metric, i.e., Hermitian metric, denoted by $($,$) . We therefore obtain a quadruple (M, J,\langle\rangle,,()$,$) , where J$ has a dual character in the sense that $(J,\langle\rangle$,$) is an almost pseudo-Hermitian structure$ on $M$ and that $(J,()$,$) is an almost Hermitian structure on M$. Thus, we have proved the following proposition.

Proposition 4. Every almost pseudo-Hermitian manifold $(M, J,\langle\rangle$,$) admits$ an almost Hermitian structure $(J,()$,$) .$

If we forget the pseudo-Hermitian metric $\langle$,$\rangle on M$ from a quadruple $(M, J,\langle$,$\rangle ,$ $()$,$) , then we obtain an almost Hermitian manifold (M, J,()$,$) . The tangent$ bundle $\xi$ of the triple $(M, J,()$,$) has the unitary group U(n)$ as the structure group, where $U(n)$ is considered as the subgroup of the orthogonal group $O(2 n)$.

Let $\nabla$ be the Riemannian connection of $(M,()$,$) . The Reimannian curvature$ tensor $R$ is given by

$$
R(X, Y) Z=\left[\nabla_{X}, \nabla_{Y}\right] Z-\nabla_{[X, Y]} Z \text { for } X, Y, Z \in \mathfrak{X}(M) .
$$

We denote by $S$ the curvature tensor of the connection $D_{X} Y=\frac{1}{2}\left(\nabla_{X} Y-J \nabla_{X} J Y\right)$;

$$
S(X, Y) Z=\left[D_{X}, D_{Y}\right] Z-D_{[X, Y]} Z \text { for } X, Y, Z \in \mathfrak{X}(M) .
$$

Associated with $S$, we obtain a complexified curvature 2-form $\Phi=\left[\Phi_{i j}\right]$ of type $(0,2)$ on the complexified tangent bundle $\xi \otimes \mathbf{C}$ with the almost Hermitian structure $(J,()$,$) as follows:$

$$
\Phi_{i j}(X, Y)=\left\langle S_{X, Y} E_{i}, E_{j}\right\rangle-\sqrt{-1}\left\langle S_{X, Y} E_{i}, J E_{j}\right\rangle \quad \text { for } X, Y \in \mathfrak{X}(M) \otimes \mathbf{C} .
$$

We can define the $k$ th Chern class $c_{k}(M)$ of $M$ to be the $k$ th Chern class $c_{k}(\xi \otimes \mathbf{C}) \in$ $H^{2 k}(M ; R)$ of $\xi \otimes \mathbf{C}$ with the almost Hermitian structure $(J,()$,$) , and the total$ Chern class of $M$ to be the sum

$$
c(M)=1+c_{1}(M)+c_{2}(M)+\cdots+c_{n}(M) \in H^{*}(M ; R) .
$$

Due to Gray [G, Theorem 9.1], the total Chern class $c(M)$ of $M$ is represented by the following $\operatorname{ad}(U(n))$-invariant polynomial:

$$
f_{n}(1, \Phi)=\left.f_{n}(\lambda, \Phi)\right|_{\lambda=1}=\left.\operatorname{det}\left(\lambda I_{n}-(2 \pi \sqrt{-1})^{-1} \Phi\right)\right|_{\lambda=1} .
$$

The Chern character of $(M, J,()$,$) , denoted by \operatorname{ch}(M)$, is defined to be the class represented by the polynomial

$$
\operatorname{trace}(\exp (-\Phi / 2 \pi \sqrt{-1})) \text {. }
$$

5. Whitney sum. We shall show that the tangent bundle $\xi$ of $(M, J,\langle\rangle$, can be reduced to a Whitney sum $\xi_{+} \otimes \xi_{-}$of a $2 p$-dimensional subbundle $\xi_{+}$with positive definite metric and a $2 q$-dimensional subbundle $\xi_{-}$with negative definite metric. The Whitney sum plays an important role in comparison between the pseudo-Chern classes and the Chern classes. 
PROPOSITION 5. $M$ admits a nonsingular field of $J$-invariant tangent $2 p$-planes and its complementary field of tangent $2 q$-planes which is also nonsingular and $J$ invariant.

PROOF. It is known (Steenrod $[\mathbf{S}, \S 40]$ ) that a compact manifold $M$ admits an indefinite metric of signature $(2 p, 2 q)$ if and only if $M$ admits a nonsingular field of tangent $2 p$-planes. $M$ also admits the complementary field of tangent $2 q$ planes. The arguments of Steenrod are based on the observation that the tangent bundle $\xi$ of $M$ admits a reduction of the structure group to $O(2 p) \times O(2 q)$, the maximal compact subgroup of $O(2 p, 2 q)$. Due to the almost complex structure $J$, the tangent bundle $\xi$ of $(M, J,\langle\rangle$,$) admits a further reduction of the structure$ group to $U(p) \times U(q)$ (as a subgroup of $O(2 p) \times O(2 q)$ ). At each point of $M$, the tangent $2 p$-plane (resp. the complementary tangent $2 q$-plane) is spanned by the vectors of positive (resp. negative) norms with respect to the metric $\langle$,$\rangle . If a$ tangent vector $u$ at a point of $M$ lies in the $2 p$-plane (resp. $2 q$-plane), then $J u$ also lies in the $2 p$-plane (resp. $2 q$-plane) since $\langle J u, J u\rangle=\langle u, u\rangle>0$ (resp. $\langle 0)$. In this sense, both fields of $2 p$-planes and of $2 q$-planes are $J$-invariant.

For the almost complex structure $J$ of a quadruple $(M, J,\langle\rangle,,()$,$) we have$

$$
J \in C^{\infty}\left(\operatorname{End}\left(\xi_{+}\right)\right) \oplus C^{\infty}\left(\operatorname{End}\left(\xi_{-}\right)\right) .
$$

Thus, we can write $J$ on $\xi_{+} \oplus \xi_{-}$as $J=J_{+}+J_{-}$, where $J_{+} \in C^{\infty}\left(\operatorname{End}\left(\xi_{+}\right)\right)$and $J_{-} \in C^{\infty}\left(\operatorname{End}\left(\xi_{-}\right)\right)$. For the algebra $\mathfrak{X}(M)$ of vector fields on $M$, we also have the splitting

$$
\mathfrak{X}(M)=\mathfrak{X}_{+}(M) \oplus \mathfrak{X}_{-}(M),
$$

where $\mathfrak{X}_{+}(M)$ (resp. $\mathfrak{X}_{-}(M)$ ) is the algebra of vector fields with values in $\xi_{+}$ (resp. $\xi_{-}$). Thus, for each $X \in \mathfrak{X}(M)$ we can write uniquely $X=X_{+}+X_{-}$, where $X_{+} \in \mathfrak{X}_{+}(M)$ and $X_{-} \in \mathfrak{X}_{-}(M)$.

Then, the almost pseudo-Hermitian metric $\langle$,$\rangle restricted to \xi_{+}$is positive definite and almost Hermitian, and similarly $\langle$,$\rangle restricted to \xi_{-}$is negative definite and almost Hermitian. Moreover, the restrictions of the almost Hermitian metric $($,$) to \xi_{+}$and $\xi_{-}$are both positive definite, and hence also almost Hermitian. Therefore, we can choose a local pseudo-orthonormal frame field $\left\{E_{a}^{+}, J_{+} E_{a}^{+} ; E_{\alpha}^{-}, J_{-} E_{\alpha}^{-}\right\}(a=1, \ldots, p ; \alpha=1, \ldots q)$ on $M$ such that $\left\{E_{a}^{+}, J_{+} E_{a}^{+}\right\}$ is a local orthonormal frame field on $\xi_{+}$with the properties

$$
\left\langle E_{a}^{+}, E_{b}^{+}\right\rangle=\left\langle J_{+} E_{a}^{+}, J_{+} E_{b}^{+}\right\rangle=\left(E_{a}^{+}, E_{b}^{+}\right)=\left(J_{+} E_{a}^{+}, J_{+} E_{b}^{+}\right)=\delta_{a b},
$$

and that $\left\{E_{\alpha}^{-}, J_{-} E_{\alpha}^{-}\right\}$is a local orthonormal frame field on $\xi_{-}$, with the properties

$$
-\left\langle E_{\alpha}^{-}, E_{\beta}^{-}\right\rangle=-\left\langle J_{-} E_{\alpha}^{-}, J_{-} E_{\beta}^{-}\right\rangle=\left(E_{\alpha}^{-}, E_{\beta}^{-}\right)=\left(J_{-} E_{\alpha}^{-}, J_{-} E_{\beta}^{-}\right)=\delta_{\alpha \beta} .
$$

We shall call such a frame the splitted basis. Then, we obtain

Proposition 6. (a) The complexified pseudo-curvature 2-form $\hat{\Phi}=\left[\hat{\Phi}_{i j}\right]$ of type $(0,2)$ on $\xi \otimes \mathbf{C}$ with $(J,\langle\rangle$,$) can be written relative to the splitted basis as a$ diagonal block matrix

with

$$
\hat{\Phi}=\left[\begin{array}{cc}
p & q \\
\hat{\Phi}_{(+)} & 0 \\
0 & \hat{\Phi}_{(-)}
\end{array}\right] p
$$

$$
{ }^{t} \hat{\Phi}_{(+)}+\overline{\hat{\Phi}}_{(+)}=0, \quad{ }^{t} \hat{\Phi}_{(-)}+\overline{\hat{\Phi}}_{(-)}=0 .
$$


(b) The complexified curvature 2 -form $\Phi=\left[\Phi_{i j}\right]$ of type $(0,2)$ on $\xi \otimes \mathbf{C}$ with $(J,()$,$) can be written relative to the splitted basis as a diagonal block matrix$

with

$$
\Phi=\left[\begin{array}{cc}
p & q \\
\Phi_{(-)} & 0 \\
0 & \Phi_{(-)}
\end{array}\right] p
$$

$$
{ }^{t} \Phi_{(+)}+\bar{\Phi}_{(+)}=0, \quad{ }^{t} \Phi_{(-)}+\bar{\Phi}_{(-)}=0 .
$$

(c) Moreover, these coincide with each other:

$$
\left[\begin{array}{cc}
\hat{\Phi}_{(+)} & 0 \\
0 & \hat{\Phi}_{(-)}
\end{array}\right]=\left[\begin{array}{cc}
\Phi_{(+)} & 0 \\
0 & \Phi_{(-)}
\end{array}\right] \text {. }
$$

PROOF. These assertions are clear from the reduction of $\xi$ to the Whitney sum $\xi_{+} \oplus \xi_{-}($see $[\mathbf{C}, \mathbf{M} \mathbf{1}])$.

The complexified pseudo-curvature 2 -form $\hat{\Phi}^{\prime}$ of type $(1,1)$ takes on $\xi_{+} \otimes \mathbf{C} \oplus$ $\xi_{-} \otimes \mathbf{C}$ with $(J,\langle\rangle$,$) the form$

$$
\hat{\Phi}^{\prime}=\left[\begin{array}{cc}
\hat{\Phi}_{(+)}^{\prime} & 0 \\
0 & \hat{\Phi}_{(-)}^{\prime}
\end{array}\right]=I_{p, q} \hat{\Phi}=\left[\begin{array}{cc}
\hat{\Phi}_{(+)} & 0 \\
0 & -\hat{\Phi}_{(-)}
\end{array}\right]=\left[\begin{array}{cc}
\Phi_{(+)} & 0 \\
0 & -\Phi_{(-)}
\end{array}\right] .
$$

The component $\Phi_{(+)}$(resp. $\Phi_{(-)}$) is the complexified curvature 2-form on $\xi_{+} \otimes \mathbf{C}$ (resp. $\left.\xi_{-} \otimes \mathbf{C}\right)$ with the structure group $U(p)$ (resp. $\left.U(q)\right)$.

Let $c_{k}\left(\xi_{+} \otimes \mathbf{C}\right)$ (resp. $\left.c_{k}\left(\xi_{-} \otimes \mathbf{C}\right)\right)$ be the $k$ th Chern class of $\xi_{+} \otimes \mathbf{C}$ (resp. $\left.\xi_{-} \otimes \mathbf{C}\right)$. Then the total Chern class

$$
c\left(\xi_{+} \otimes \mathbf{C}\right)=1+c_{1}\left(\xi_{+} \otimes \mathbf{C}\right)+c_{2}\left(\xi_{+} \otimes \mathbf{C}\right)+\cdots+c_{p}\left(\xi_{+} \otimes \mathbf{C}\right)
$$

of $\xi_{+} \otimes \mathbf{C}$ is represented by

$$
f_{p}\left(1, \Phi_{(+)}\right)=\operatorname{det}\left(I_{p}-(2 \pi \sqrt{-1})^{-1} \Phi_{(+)}\right) .
$$

Similarly, the total Chern class

$$
c\left(\xi_{-} \otimes \mathbf{C}\right)=1+c_{1}\left(\xi_{-} \otimes \mathbf{C}\right)+c_{2}\left(\xi_{-} \otimes \mathbf{C}\right)+\cdots+c_{q}\left(\xi_{-} \otimes \mathbf{C}\right)
$$

of $\xi_{-} \otimes \mathbf{C}$ is represented by

$$
f_{q}\left(1, \Phi_{(-)}\right)=\operatorname{det}\left(I_{q}-(2 \pi \sqrt{-1})^{-1} \Phi_{(-)}\right) .
$$

Then the total Chern class $c(M)$ of $(M, J,()$,$) represented by (2)$ is determined in terms of $c\left(\xi_{+} \otimes \mathbf{C}\right)$ and $c\left(\xi_{-} \otimes \mathbf{C}\right)$ by the formula

$$
\begin{aligned}
c(M)= & c\left(\xi_{+} \otimes \mathbf{C}\right) c\left(\xi_{-} \otimes \mathbf{C}\right) \\
= & \left(1+c_{1}\left(\xi_{+} \otimes \mathbf{C}\right)+c_{2}\left(\xi_{+} \otimes \mathbf{C}\right)+\cdots+c_{p}\left(\xi_{+} \otimes \mathbf{C}\right)\right) \\
& \cdot\left(1+c_{1}\left(\xi_{-} \otimes \mathbf{C}\right)+c_{2}\left(\xi_{-} \otimes \mathbf{C}\right)+\cdots+c_{q}\left(\xi_{-} \otimes \mathbf{C}\right)\right) .
\end{aligned}
$$

Inserting $\Phi$ of the form obtained in Proposition 6(b) into (2), we can easily see this.

For the Chern character $\operatorname{ch}(M)$ of $(M, J()$,$) , we have a well-known formula$

$$
\operatorname{ch}(M)=\operatorname{ch}\left(\xi_{+} \otimes \mathbf{C}\right)+\operatorname{ch}\left(\xi_{-} \otimes \mathbf{C}\right)
$$

where $\operatorname{ch}\left(\xi_{ \pm} \otimes \mathbf{C}\right)$ are the Chern characters of $\xi_{ \pm} \otimes \mathbf{C}$ represented by

$$
\operatorname{trace}\left(\exp \left(-\Phi_{( \pm)} / 2 \pi \sqrt{-1}\right)\right)
$$

6. Pseudo-Chern classes and Chern classes. In this section, we shall establish a relation between the pseudo-Chern classes and Chern classes. We now state the main result. 
THEOREM 7. The total pseudo-Chern class $\hat{c}(M)$ of an almost pseudoHermitian manifold $(M, J,\langle\rangle$,$) is determined by the total Chern class c\left(\xi_{+} \otimes \mathbf{C}\right)$ of $\xi_{+} \otimes \mathbf{C}$ and the total Chern class $c\left(\overline{\xi_{-} \otimes \mathbf{C}}\right)$ of the conjugate bundle $\overline{\xi_{-} \otimes \mathbf{C}}$ of $\xi_{-} \otimes \mathbf{C}$ through the formula

$$
\hat{c}(M)=c\left(\xi_{+} \otimes \mathbf{C}\right) c\left(\overline{\xi_{-} \otimes \mathbf{C}}\right) .
$$

ProOF. From Definition 2, the total pseudo-Chern class $\hat{c}(M)$ is represented by $\hat{f}(1, \hat{\Phi})$. From Proposition 6 , we can write $\hat{\Phi}$ as the diagonal block matrix relative to the splitted basis. Inserting such a form $\hat{\Phi}$ into $\hat{f}(1, \hat{\Phi})$, we have

$$
\begin{aligned}
\hat{f}(1, \hat{\Phi})= & (-1)^{q} \operatorname{det}\left(I_{p, q}-(2 \pi \sqrt{-1})^{-1} \hat{\Phi}\right) \\
= & (-1)^{q} \operatorname{det}\left(I_{p}-(2 \pi \sqrt{-1})^{-1} \Phi_{(+)}\right) \\
& \wedge \operatorname{det}\left(-I_{q}-(2 \pi \sqrt{-1})^{-1} \Phi_{(-)}\right) \\
= & (-1)^{q} \operatorname{det}\left(I_{p}-(2 \pi \sqrt{-1})^{-1} \Phi_{(+)}\right) \\
& \wedge \operatorname{det}^{t}\left(-I_{q}-(2 \pi \sqrt{-1})^{-1} \Phi_{(-)}\right) \\
= & (-1)^{q} \operatorname{det}\left(I_{p}-(2 \pi \sqrt{-1})^{-1} \Phi_{(+)}\right) \\
& \wedge\left(-I_{q}+(2 \pi \sqrt{-1})^{-1} \bar{\Phi}_{(-)}\right),
\end{aligned}
$$

where the last equality holds since ${ }^{t} \Phi_{(-)}=-\bar{\Phi}_{(-)}$. For the second term of the above expression, we have

$$
\begin{aligned}
\operatorname{det}\left(-I_{q}+(2 \pi \sqrt{-1})^{-1} \bar{\Phi}_{(-)}\right) & =-\operatorname{det}\left(I_{q}-(2 \pi \sqrt{-1})^{-1} \bar{\Phi}_{(-)}\right) \quad \text { if } q \text { is odd } \\
& =\operatorname{det}\left(I_{q}-(2 \pi \sqrt{-1})^{-1} \bar{\Phi}_{(-)}\right) \quad \text { if } q \text { is even. }
\end{aligned}
$$

Therefore, in any case, we have

$$
\begin{aligned}
\hat{f}(1, \hat{\Phi}) & =\operatorname{det}\left(I_{p}-(2 \pi \sqrt{-1})^{-1} \Phi_{(+)}\right) \wedge \operatorname{det}\left(I_{q}-(2 \pi \sqrt{-1})^{-1} \bar{\Phi}_{(-)}\right) \\
& =f_{p}\left(1, \Phi_{(+)}\right) \wedge f_{q}\left(1, \bar{\Phi}_{(-)}\right) .
\end{aligned}
$$

The first term of the right-hand side clearly represents the total Chern class $c\left(\xi_{+} \otimes \mathbf{C}\right)$ of the complexified bundle $\xi_{+} \otimes \mathbf{C}$. Since $\overline{\boldsymbol{\Phi}}_{(-)}$is considered as the curvature 2 -form of the conjugate bundle $\overline{\xi_{-} \otimes \mathbf{C}}$, the polynomial

$$
f_{q}\left(1, \bar{\Phi}_{(-)}\right)=\operatorname{det}\left(I_{q}-(2 \pi \sqrt{-1})^{-1} \bar{\Phi}_{(-)}\right)
$$

represents the total Chern class $c\left(\overline{\xi_{-} \otimes \mathbf{C}}\right)$ of $\overline{\xi_{-} \otimes \mathbf{C}}$.

Therefore, if $\xi$ - is flat, then the total pseudo-Chern class $\hat{c}(M)$ coincides with the total Chern class $c(M)$.

Since the $k$ th Chern class $c_{k}\left(\overline{\xi_{-} \otimes \mathbf{C}}\right)$ is equal to $(-1)^{k} c_{k}\left(\xi_{-} \otimes \mathbf{C}\right)$ [M-S, Lemma 14.9], the total pseudo-Chern class $\hat{c}(M)$ is expressed in terms of the Chern classes $c_{i}\left(\xi_{+} \otimes \mathbf{C}\right)$ and $c_{j}\left(\xi_{-} \otimes \mathbf{C}\right)$ as follows:

$$
\begin{aligned}
\hat{c}(M)= & \left(1+c_{1}\left(\xi_{+} \otimes \mathbf{C}\right)+c_{2}\left(\xi_{+} \otimes \mathbf{C}\right)+\cdots+c_{p}\left(\xi_{+} \otimes \mathbf{C}\right)\right) \\
& \cdot\left(1-c_{1}\left(\xi_{-} \otimes \mathbf{C}\right)+c_{2}\left(\xi_{-} \otimes \mathbf{C}\right)-\cdots+(-1)^{q} c_{q}\left(\xi_{-} \otimes \mathbf{C}\right)\right) .
\end{aligned}
$$

From this formula, we have useful expressions for the pseudo-Chern class as follows. 
Proposition 8. The kth pseudo-Chern class $\hat{c}_{k}(M)$ of $(M, J,\langle\rangle$,$) is deter-$ mined by the Chern classes $c_{i}\left(\xi_{+} \otimes \mathbf{C}\right)$ and $c_{j}\left(\xi_{-} \otimes \mathbf{C}\right)$ as follows:

(i) for $p \geq q \geq k$,

$$
\begin{aligned}
\hat{c}_{k}(M)= & c_{k}\left(\xi_{+} \otimes \mathbf{C}\right)-c_{k-1}\left(\xi_{+} \otimes \mathbf{C}\right) c_{1}\left(\xi_{-} \otimes \mathbf{C}\right) \\
& +c_{k-2}\left(\xi_{+} \otimes \mathbf{C}\right) c_{2}\left(\xi_{-} \otimes \mathbf{C}\right)-\cdots+(-1)^{k} c_{k}\left(\xi_{-} \otimes \mathbf{C}\right),
\end{aligned}
$$

(ii) for $p \geq k \geq q$,

$$
\begin{aligned}
\hat{c}_{k}(M)= & c_{k}\left(\xi_{+} \otimes \mathbf{C}\right)-c_{k-1}\left(\xi_{+} \otimes \mathbf{C}\right) c_{1}\left(\xi_{-} \otimes \mathbf{C}\right) \\
& +c_{k-2}\left(\xi_{+} \otimes \mathbf{C}\right) c_{2}\left(\xi_{-} \otimes \mathbf{C}\right)-\cdots+(-1)^{q} c_{k-q}\left(\xi_{+} \otimes \mathbf{C}\right) c_{q}\left(\xi_{-} \otimes \mathbf{C}\right),
\end{aligned}
$$

(iii) for $n=p+q \geq k \geq p \geq q$,

$$
\begin{aligned}
\hat{c}_{k}(M)= & (-1)^{k-p} c_{p}\left(\xi_{+} \otimes \mathbf{C}\right) c_{k-p}\left(\xi_{-} \otimes \mathbf{C}\right) \\
& +(-1)^{k-p+1} c_{p-1}\left(\xi_{+} \otimes \mathbf{C}\right) c_{k-p+1}\left(\xi_{-} \otimes \mathbf{C}\right) \\
& +(-1)^{k-p+2} c_{p-2}\left(\xi_{+} \otimes \mathbf{C}\right) c_{k-p+2}\left(\xi_{-} \otimes \mathbf{C}\right) \\
& \cdots \\
& +(-1)^{q} c_{k-q}\left(\xi_{+} \otimes \mathbf{C}\right) c_{q}\left(\xi_{-} \otimes \mathbf{C}\right) .
\end{aligned}
$$

Concerning the top pseudo-Chern class, we have

Proposition 9. (i) $\hat{c}_{n}(M)=(-1)^{q} c_{n}(M)$. (ii) $\hat{c}_{n}(M)=(-1)^{p} e(\xi)^{2}(e(\xi)$ : the Euler class of $\xi)$. have

PROOF. (i) From the third formula in Proposition 8, with $k=n=p+q$, we

$$
\hat{c}_{n}(M)=(-1)^{q} c_{p}\left(\xi_{+} \otimes \mathbf{C}\right) c_{q}\left(\xi_{-} \otimes \mathbf{C}\right),
$$

where $c_{p}\left(\xi_{+} \otimes \mathbf{C}\right) c_{q}\left(\xi_{-} \otimes \mathbf{C}\right)$ is equal to the top Chern class $c_{n}(M)$.

(ii) It is known that the top Chern class $c_{n}(M)=c_{n}(\xi \otimes \mathbf{C})$ is equal to the Euler class $e\left((\xi \otimes \mathbf{C})_{R}\right)$ of the underlying real vector bundle $(\xi \otimes \mathbf{C})_{R}$ of $\xi \otimes \mathbf{C}$. Moreover, there is a relation between $e\left((\xi \otimes \mathbf{C})_{R}\right)$ and the Euler class $e(\xi \oplus \xi)$ of the Whitney sum $\xi \oplus \xi$ as follows $[\mathbf{M}-\mathbf{S}, \S 15.7,8]$ :

$$
e\left((\xi \otimes \mathbf{C})_{R}\right)=(-1)^{n} e(\xi \oplus \xi) .
$$

Since $e(\xi \oplus \xi)=e(\xi)^{2}$, together with i), we have

$$
\hat{c}_{n}(M)=(-1)^{q} c_{n}(M)=(-1)^{n+q} e(\xi)^{2}=(-1)^{p} e(\xi)^{2} .
$$

This completes the proof.

We now turn our attention to the pseudo-Chern character. Corresponding to the formula (5), we have

Proposition 10. The pseudo-Chern character $\widehat{\operatorname{ch}}(M)$ of $(M, J,\langle\rangle$,$) is deter-$ mined by the Chern characters $\operatorname{ch}\left(\xi_{+} \otimes \mathbf{C}\right)$ and $\operatorname{ch}\left(\overline{\xi_{-} \otimes \mathbf{C}}\right)$ as follows:

$$
\widehat{\operatorname{ch}}(M)=\operatorname{ch}\left(\xi_{+} \otimes \mathbf{C}\right)+\operatorname{ch}\left(\overline{\xi_{-} \otimes \mathbf{C}}\right) .
$$

Proof. From Definition 3, the pseudo-Chern character $\widehat{c h}(M)$ is represented by the polynomial

$$
\operatorname{trace}\left(\exp \left(-\hat{\Phi}^{\prime} / 2 \pi \sqrt{-1}\right)\right)
$$


Since $\hat{\Phi}^{\prime}$ takes the form (3) on the Whitney sum $\xi_{+} \otimes \mathbf{C} \oplus \xi_{-} \otimes \mathbf{C}$ with $(J,\langle\rangle$,$) ,$ we have

$$
\begin{aligned}
\operatorname{trace} & \left(\exp \left(-\hat{\Phi}^{\prime} / 2 \pi \sqrt{-1}\right)\right) \\
& =\operatorname{trace}\left(\exp \left[\begin{array}{cc}
-\Phi_{(+)} / 2 \pi \sqrt{-1} & 0 \\
0 & \Phi_{(-)} / 2 \pi \sqrt{-1}
\end{array}\right]\right) \\
& =\operatorname{trace}\left[\begin{array}{cc}
\exp \left(-\Phi_{(+)} / 2 \pi \sqrt{-1}\right) & 0 \\
0 & \exp \left(\Phi_{(-)} / 2 \pi \sqrt{-1}\right)
\end{array}\right] \\
& =\operatorname{trace}\left(\exp \left(-\Phi_{(+)} / 2 \pi \sqrt{-1}\right)\right)+\operatorname{trace}\left(\exp \left(\Phi_{(-)} / 2 \pi \sqrt{-1}\right)\right) .
\end{aligned}
$$

The first term represents the Chern character $\operatorname{ch}\left(\xi_{+} \otimes \mathbf{C}\right)$. Concerning the second term, further we have

$$
\begin{aligned}
\operatorname{trace}\left(\exp \left(\Phi_{(-)} / 2 \pi \sqrt{-1}\right)\right. & =\operatorname{trace}\left({ }^{t}\left(\exp \left(\Phi_{(-)} / 2 \pi \sqrt{-1}\right)\right)\right) \\
& =\operatorname{trace}\left(\exp \left({ }^{t} \Phi_{(-)} / 2 \pi \sqrt{-1}\right)\right) \\
& =\operatorname{trace}\left(\exp \left(-\bar{\Phi}_{(-)} / 2 \pi \sqrt{-1}\right)\right) .
\end{aligned}
$$

The last expression represents the Chern character of $\overline{\xi_{-} \otimes \mathbf{C}}$. This completes the proof.

Proposition 11. The difference between $\widehat{\operatorname{ch}}(M)$ and $\operatorname{ch}(M)$ depends only on the complexified subbundle $\xi_{-} \otimes \mathbf{C}$ :

$$
\widehat{\operatorname{ch}}(M)-\operatorname{ch}(M)=\operatorname{ch}\left(\overline{\xi_{-} \otimes \mathbf{C}}\right)-\operatorname{ch}\left(\xi_{-} \otimes \mathbf{C}\right) .
$$

ProOF. This relation is easily verified from Proposition 10 and (5).

If $\xi_{-}$is flat, then we have the coincidence $\widehat{\operatorname{ch}}(M)=\operatorname{ch}(M)$.

Let $-\langle$,$\rangle denote an indefinite metric \langle$,$\rangle with the opposite signature$

$$
(-\cdots-+\cdots+), \quad 2 p \geqq 2 q, \quad 2 p+2 q=2 n .
$$

A manifold carrying an indefinite metric $\langle$,$\rangle clearly admits the opposite one -\langle$,$\rangle .$

Associated with $(M, J,\langle\rangle$,$) , we now consider an almost pseudo-Hermitian man-$ ifold $(M, J,-\langle\rangle$,$) . The metric -\langle$,$\rangle is negative definite and almost Hermitian$ on the $2 p$-dimensional subbundle $\xi_{+}$, and is positive definite and almost Hermitian on the $2 q$-dimensional subbundle $\xi_{-}$. According to Definition 2 , the total pseudo-Chern class of $(M, J,-\langle\rangle$,$) , denoted by \hat{c}_{-}(M)$, is represented by

$$
(-1)^{p} \operatorname{det}\left(-I_{p, q}-(2 \pi \sqrt{-1})^{-1} \hat{\Phi}\right) \text {. }
$$

From this polynomial, we can obtain a formula analogous to that in Theorem 7 as follows:

$$
\hat{c}_{-}(M)=c\left(\overline{\xi_{+} \otimes \mathbf{C}}\right) c\left(\xi_{-} \otimes \mathbf{C}\right) .
$$

If we denote by $\widehat{c h}_{-}(M)$ the pseudo-Chern character of $(M, J,-\langle\rangle$,$) , then we also$ have a formula corresponding to that in Proposition 10 as follows:

$$
\widehat{c h}_{-}(M)=\operatorname{ch}\left(\overline{\xi_{+} \otimes \mathbf{C}}\right)+\operatorname{ch}\left(\xi_{-} \otimes \mathbf{C}\right) .
$$

Moreover, the difference between $\widehat{c h}_{-}(M)$ and $\operatorname{ch}(M)$ depends only on $\xi_{+} \otimes \mathbf{C}$ (cf. Proposition 11):

$$
\widehat{c h}_{-}(M)-\operatorname{ch}(M)=\operatorname{ch}\left(\overline{\xi_{+} \otimes \mathbf{C}}\right)-\operatorname{ch}\left(\xi_{+} \otimes \mathbf{C}\right) .
$$


If the negative definite part $\xi_{+}$is flat, then we have the coincidences

$$
\hat{c}_{-}(M)=c(M) \text { and } \widehat{c h}_{-}(M)=\operatorname{ch}(M) .
$$

Thus, the pseudo-Chern classes and the pseudo-Chern character are not invariant by an overall change of sign of the metric.

We may therefore say that the pseudo-Chern classes and also the pseudo-Chern character of $(M, J,\langle\rangle$,$) are considered as the characteristic classes which charac-$

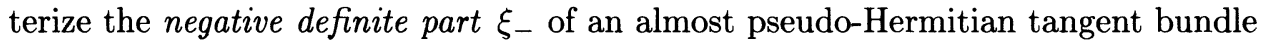
$\xi \cong \xi_{+} \oplus \xi_{-}$.

7. Remarks. We end this paper with some remarks.

A. We now explain why we can construct the pseudo-Chern classes in addition to the Chern classes on the almost pseudo-Hermitian manifold $M$. The complexification $\xi \otimes \mathbf{C}$ of the tangent bundle $\xi$ splits into a Whitney sum $\xi_{+} \otimes \mathbf{C} \oplus \xi_{-} \otimes \mathbf{C}$. Thus, we can construct four kinds of $\operatorname{ad}(U(p) \times U(q))$-invariant curvature forms corresponding to the following bundles:

$$
\xi_{+} \otimes \mathbf{C} \oplus \xi_{-} \otimes \mathbf{C}, \quad \xi_{+} \otimes \mathbf{C} \oplus \overline{\xi_{-} \otimes \mathbf{C}}, \quad \overline{\xi_{+} \otimes \mathbf{C}} \oplus \overline{\xi_{-} \otimes \mathbf{C}}, \quad \overline{\xi_{+} \otimes \mathbf{C}} \oplus \xi_{-} \otimes \mathbf{C} .
$$

By an overall conjugation, we can make the third and the fourth bundles coincide with the first and the second bundles, respectively. Therefore, we have two essentially different characteristic classes up to conjugation:

$$
c\left(\xi_{+} \otimes \mathbf{C} \oplus \xi_{-} \otimes \mathbf{C}\right), \quad c\left(\xi_{+} \otimes \mathbf{C} \oplus \overline{\xi_{-} \otimes \mathbf{C}}\right) .
$$

The former is the total Chern class considered by Borel $[\mathbf{B}]$, and the latter is the total pseudo-Chern class constructed in this paper.

B1. A 4-dimensional almost pseudo-Hermitian manifold $(M, J,\langle\rangle$,$) of signature$ $(2 p, 2 q)=(2,2)$, with $U(1,1)$ as the structure group, is interesting for the following reasons. (1) It is a lowest-dimensional example of an almost pseudo-Hermitian manifold. (2) Every 4-dimensional pseudo-Riemannian manifold of metric signature $(2,2)$ admits an almost complex structure. Here, we know that an orientable pseudo-Riemannian 4-manifold of signature $(2,2)$ admits a field of nonsingular oriented 2-planes $[\mathbf{S}, \S 40 ; \mathbf{M 2}$, Lemma A], and thanks to Hirzebruch and Hopf $[\mathbf{H}-\mathbf{H}$, $4.5,6]$ that it also possesses an almost complex structure.

B2. A simple and illustrative example may be a product space $M=\mathbf{C} P^{1} \times \mathbf{C} P^{1}$ of two projective spaces of complex dimension 1. Let $\mathbf{C} P_{(i)}^{1}(i=1,2)$ denote the $i$ th component $\mathbf{C} P^{1}$ of $M$, and $(,)_{i}$ and $J_{i}$ be the standard Hermitian metric and the complex structure on $\mathbf{C} P_{(i)}^{1}$, respectively. Then, we have a 4-dimensional (almost) pseudo-Hermitian manifold $(M, J,\langle\rangle,,()$,$) , where J=J_{1}+J_{2},\langle\rangle=,(,)_{1}-(,)_{2}$, and $()=,(,)_{1}+(,)_{2} \cdot \mathbf{C} P_{(i)}^{1}$ has the first Chern class $c_{1}\left(\mathbf{C} P_{(i)}^{1}\right)=2 a_{i}$ such that the Kronecker index $\left\langle a_{i}, \mu_{i}\right\rangle=1$, where $a_{i}$ and $\mu_{i}$ are a suitably chosen generator for $H^{2}\left(\mathbf{C} P_{(i)}^{1} ; Z\right)$ and the fundamental homology class of $\mathbf{C} P_{(i)}^{1}$, respectively. We have the Chern classes and the pseudo-Chern classes as follows:

$$
\begin{array}{ll}
c_{1}(M)=2 a_{1}+2 a_{2}, & \hat{c}_{1}(M)=2 a_{1}-2 a_{2}, \\
c_{2}(M)=4 a_{1} a_{2}, & \hat{c}_{2}(M)=-4 a_{1} a_{2} .
\end{array}
$$

Concerning the pseudo-Chern numbers, we have, for example,

$$
\begin{aligned}
\hat{c}_{1}(M)^{2}[M] & =\left\langle\left(2 a_{1}-2 a_{2}\right)^{2}, \mu_{1} \times \mu_{2}\right\rangle \\
& =-8\left\langle a_{1}, \mu_{1}\right\rangle\left\langle a_{2}, \mu_{2}\right\rangle=-8 .
\end{aligned}
$$


B3. For a 4-dimensional almost complex (or Hermitian) manifold, Wu's relation $[\mathbf{W}]$ is known as follows:

$$
p_{1}(M)+2 \chi(M)=c_{1}(M)^{2},
$$

where $p_{1}(M)$ is the first Pontrjagin class, $\chi(M)$ the Euler class and $c_{1}(M)$ the first Chern class. If $M$ is a 4-dimensional almost pseudo-Hermitian manifold, then both $p_{1}(M)$ and $\chi(M)$ in the above relation can be determined by its underlying real pseudo-Riemannian structure (see $[\mathbf{M 1}]$ for $p_{1}(M)$ and $[\mathbf{A}, \mathbf{C}]$ for $\chi(M)$ ). However, $c_{1}(M)$ in the right-hand side cannot be replaced by the first pseudo-Chern class $\hat{c}_{1}(M)$.

C. It is not hard to see the coincidence of the pseudo-Pontrjagin classes with the Pontrjagin classes in terms of the pseudo-Chern classes, which is an alternative proof of the main theorem in $[\mathbf{M 1}]$.

D. The pseudo-Chern numbers do not in general coincide with the Chern numbers (cf. B2 above).

E1. The condition $[\mathbf{S}, \S 40]$ for a manifold to possess a pseudo-Riemannian metric is rather restrictive. Consider, for example, whether a complex projective space C $P^{4 m-2}$ of complex dimension $4 m-2$ admits a reduction of the structure group to $U(p, 4 m-p-2)$, with $p=1 \bmod 2$. Suppose that it admits such a structure. Then its underlying real manifold has an indefinite metric of signature $(2 p, 8 m-$ $2 p-4)(2 p=2 \bmod 4)$, and hence from an earlier result [M3, Proposition 9] its Euler characteristic $\chi$ is even and is congruent modulo 4 to the Hirzebruch index $\tau$. However, $\chi\left(\mathbf{C} P^{4 m-2}\right)=4 m-1$, and moreover $\chi\left(\mathbf{C} P^{4 m-2}\right)-\tau\left(\mathbf{C} P^{4 m-2}\right)=$ $4 m-2 \neq 0 \bmod 4$, which is a contradiction. Thus, $\mathbf{C} P^{4 m-2}$ does not admit such a structure.

E2. Since the complex projective spaces are the special cases of the complex Grassmann manifolds, the above example suggests that we must take care about which types of signature for the metrics can be admitted on the complex Grassmann manifolds.

F. However, there are many nontrivial examples of (almost) pseudo-Hermitian manifolds, or more specifically, pseudo-Hermitian symmetric spaces, pseudo-Kähler manifolds, etc. The complex Heisenberg manifold is an interesting example that is pseudo-Kähler but not Kähler, and whose Chern classes and pseudo-Chern classes are all zero. We will develop further discussions on theses manifolds elsewhere.

ACKNowledgments. I would like to thank Professor C. Ihara, Professor S. Takizawa, Professor K. Sekigawa, Dr. T. Iwai and Dr. T. Higa for conversations and helpful advice. I also wish to thank the referee for valuable comments and for calling my attention to the complex Heisenberg manifold, the other nontrivial examples, and an important article of Borel.

\section{REFERENCES}

[A] A. Avez, Formule de Gauss-Bonnet-Chern en métrique de signature quelconque, C. R. Acad. Sci. Paris 255 (1962), 2049-2051.

[B] A. Borel, Sur une généralisation de la formule de Gauss-Bonnet, An. Acad. Brasil. Ci. 39 (1969), 31-37.

[C] S. S. Chern, Pseudo-Riemannian geometry and the Gauss-Bonnet formula, An. Acad. Brasil. Ci. 35 (1963), 17-26.

[G] A. Gray, Nearly Kähler manifolds, J. Differential Geometry 4 (1970), 283-309. 
[H-H] F. Hirzebruch and H. Hopf, Felder von Flächenelementen in 4-dimensionalen Mannigfaltigkeiten, Math. Ann. 136 (1958), 156-172.

[K-N] S. Kobayashi and K. Nomizu, Foundations of differential geometry, vol. II, Interscience, New York, 1969.

[M1] Y. Matsushita, Pseudo-Pontrjagin classes, Proc. Amer. Math. Soc. 93 (1985), 521-524.

[M2] _ , On Euler characteristics of compact Einstein 4-manifolds of metric signature (+ + - ), J. Math. Phys. 22 (1981), 979-982.

[M3] - Thorpe-Hitchin inequality for compact Einstein 4-manifolds of metric signature $(++--)$ and the generalized Hirzebruch index formula, J. Math. Phys. 24 (1983), 36-40.

[M-S] J. Milnor and J. Stasheff, Characteristic classes, Ann. of Math. Studies, No. 76, Princeton Univ. Press, Princeton, N. J., 1974

[S] N. Steenrod, The topology of fibre bundles, Princeton Univ. Press., Princeton, N.J., 1951.

[W] W. T. Wu, Sur la structure presque complexe d'une variété différentiable réelle de dimension $4, \mathrm{C} . \mathrm{R}$. Acad. Sci. Paris 227 (1948), 1076-1078.

Department of Applied Mathematics and Physics , FACUlty of EngineERING, KYOTO UNIVERSITY, KYOTO 606, JAPAN 